\title{
Corrigendum: Enhanced role of eddies in the Arctic marine biological pump
}

\author{
Eiji Watanabe, Jonaotaro Onodera, Naomi Harada, Makio C. Honda, Katsunori Kimoto, Takashi Kikuchi, \\ Shigeto Nishino, Kohei Matsuno, Atsushi Yamaguchi, Akio Ishida \& Michio J. Kishi
}

Nature Communications 5:3950 doi: 10.1038/ncomms4950 (2014); Published 27 May 2014; Updated 30 Sep 2015

During the analysis of additional data, the authors identified errors in the original Article. The first relates to the presentation of sinking particle flux data in Fig. 2c, which was mistakenly plotted in reference to a previous dataset, rather than Supplementary Data 1. The corrected version of Fig. 2 appears below. The second error concerns the miscalculation of the Particulate Organic Nitrogen (PON) and Carbon (POC) fluxes presented in Supplementary Data 1 and Fig. 3b,c. During the flux calculation process, we mistakenly used the weight of analysed subsamples rather than aliquot samples, which resulted in an underestimation of the mole fluxes of PON and POC in the published Article. Two new columns, detailing the correctly calculated fluxes, have been added to Supplementary Data 1, while the corrected version of Fig. 3 appears below.

As a consequence of these corrections, the Article text in the Results section requires further revisions: the description of the POC and PON fluxes in the Results section should cite the revised values 926.9 and $94.0 \mu \mathrm{mol}-\mathrm{Nm}^{-2}$ per day, rather than 124.5 and $12.6 \mu \mathrm{mol}-\mathrm{Nm}^{-2}$ per day; and the comparison of observed and modelled should state that modelled fluxes better reflect trap data at the shallower, rather than the deeper site as was originally stated.

Finally, an additional typographical correction must be applied to the text in the Results scetion. The sixth sentence should refer to 'November 2010', rather than 'November 2011'.

We sincerely apologise for these errors and the corresponding confusion. We assure the reader that these corrections in no way alter the conclusions in the published article. 


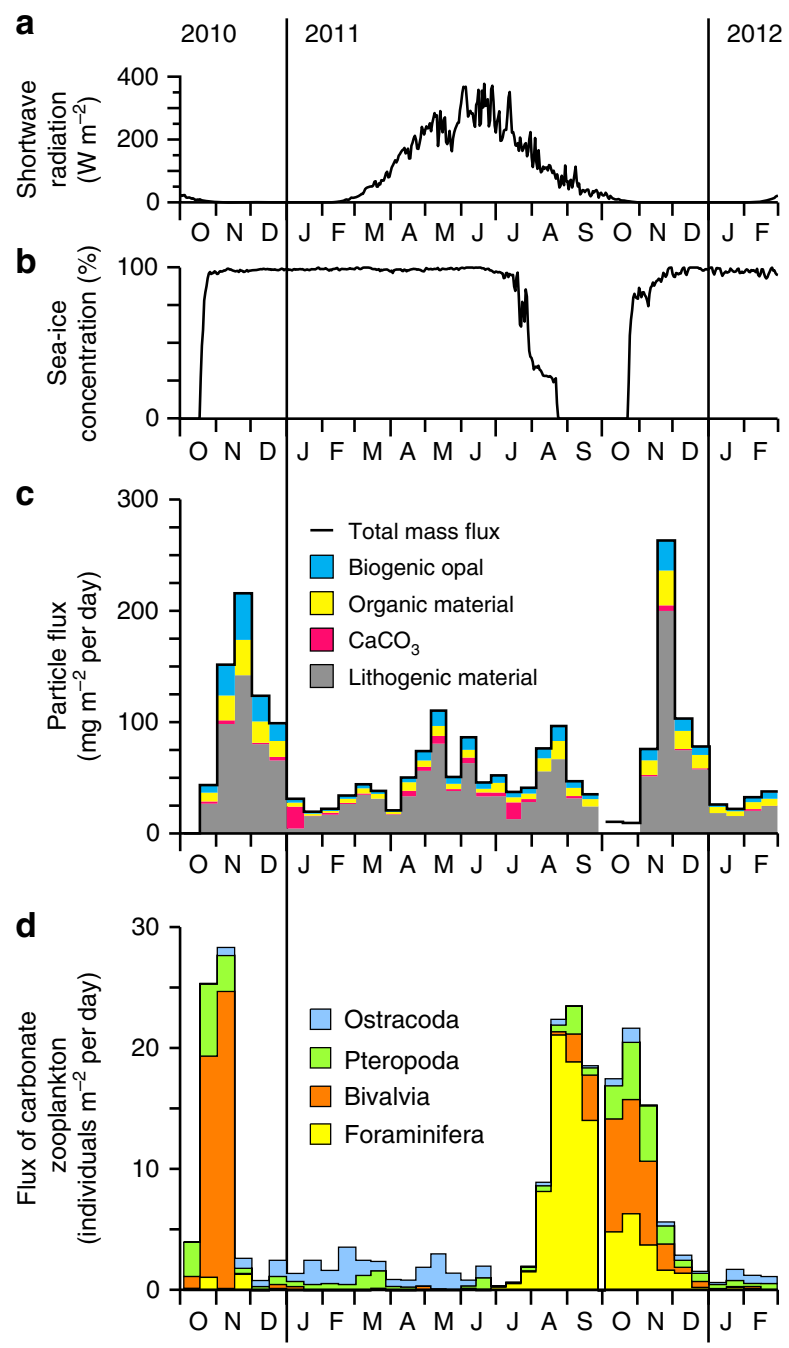

Figure 2. 

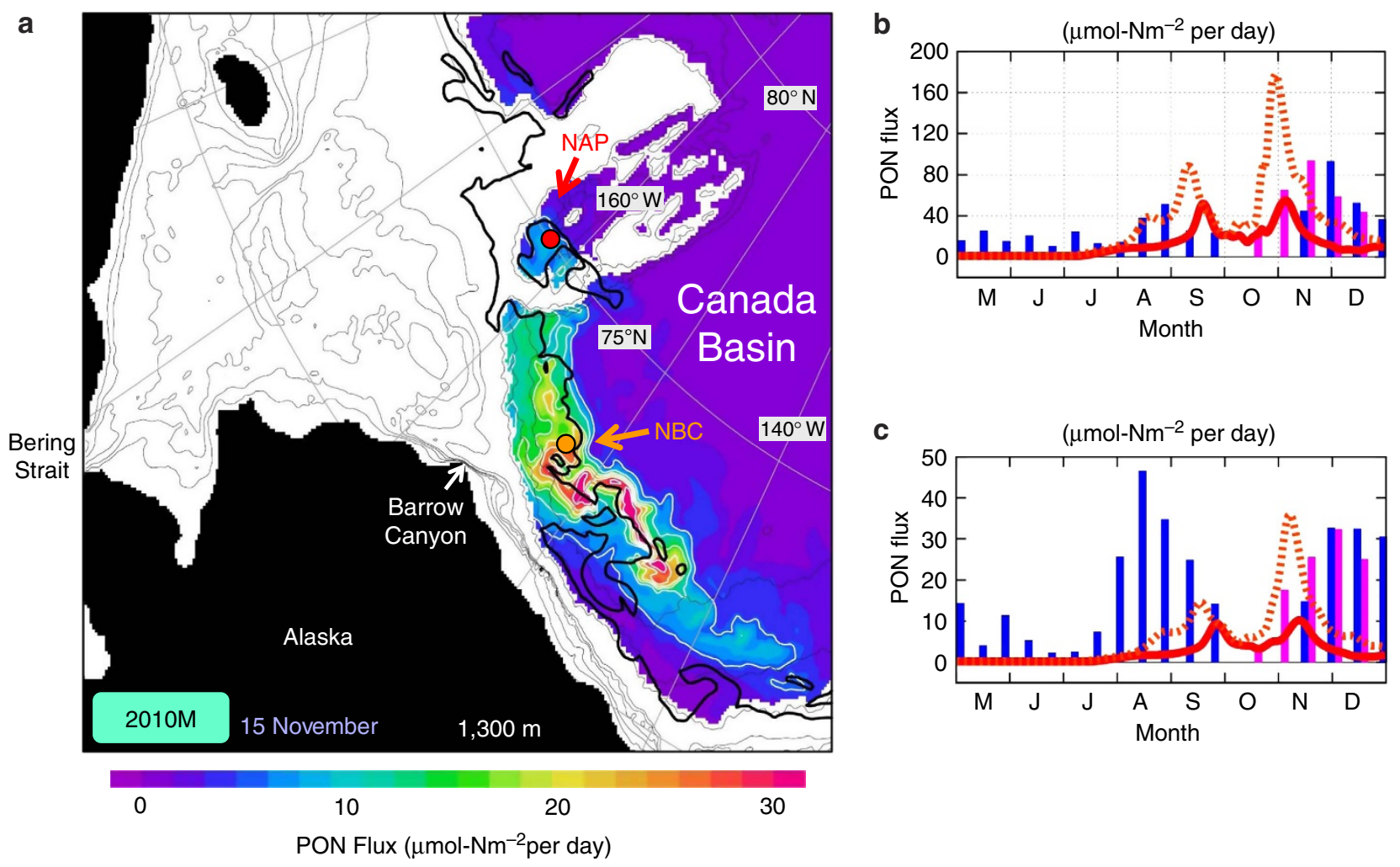

Figure 3.

This work is licensed under a Creative Commons Attribution 4.0 International License. The images or other third party material in this article are included in the cc. to obtain permission from the license holder to reproduce the material. To view a copy of this license, visit http://creativecommons.org/licenses/nd/4.0/ 